\title{
Revisão para psicólogos da segunda causa mais comum de demência neurodegenerativa em idosos
}

\author{
R. Bertelli, J.J.P. Bianchi, E.C. Cruz
}

Formas perniciosas de declínio cognitivo são causadas por uma variedade de condições neuropatológicas. Entre as causas das condições de declínio cognitivo pernicioso está a Demência de Corpos de Lewy (DCL). Apesar de representar a segunda causa mais comum de demência neurodegenerativa em idosos, é ainda pouco conhecida pelos psicólogos. Os psicólogos podem contribuir de forma significativa no processo de avaliação cognitiva e de diagnóstico diferencial entre essa condição neurodegenerativa e condições neuropsiquiátricas. O conhecimento da DCL é, portanto, extremamente importante e pode significar uma diminuição na taxa de mortalidade.

Palavras-chave: demência de corpos de Lewy, declínio cognitivo, avaliação cognitiva

\section{Abbreviated review for psychologists of the second most common cause of neurodegenerative disease in elderly people}

Malignant forms of cognitive decline are caused by a variety of neuropathological conditions. Dementia with Lewy bodies (DLB) is among such conditions. Even though such a condition represents the second most frequent cause of neurodegenerative dementia in elderly people, it is still very little known by psychologists. Psychologists can play a very important role in the process of cognitive evaluation and differential diagnosis between this neurodegenerative condition and neuropsychiatric conditions. Knowledge of DLB is thus extremely important and it may mean a decrease in fatality rate.

Key words: dementia with Lewy bodies, cognitive decline, cognitive evaluation

Rosangela Bertelli. Centro de Investigação em Desporto, Saúde e Desenvolvimento Humano e Universidade de Trás-os-Montes e Alto Douro.

José João Pinhanços de Bianchi e Eduardo Chaves Cruz. Universidade de Trás-os-Montes e Alto Douro. Endereço para correspondência: Rosangela Bertelli, Departamento de Educação e Psicologia, Edifício do CIFOP, Rua Dr. Manuel Cardona, 5001-558 Vila Real - Portugal.E-mail: bertelli@utad.pt 
A revisão que aqui se apresenta pretende oferecer uma panorâmica básica, que se julga ser especialmente útil para psicólogos, os quais podem desempenhar um papel fundamental no processo de diagnóstico da Demência de Corpos de Lewy $(D C L)$ e na elaboração e realização de programas de reabilitação cognitiva, que auxiliem o paciente de DCL a agir, o mais adequadamente possível, dentro das suas contingências operantes (Bertelli, Vasconcelos-Raposo, \& Fernandes, 2007; Salmon \& Bondi, 2009).

Envelhecer não é sinónimo de incapacidade cognitiva. Cada indivíduo tem uma história pessoal, o que lhe confere uma certa individualidade, ou seja, indivíduos diferentes envelhecem de modos diferentes. É verdade que, no processo de envelhecimento, muitos indivíduos manifestam algum declínio em certas habilidades cognitivas, porém muitos outros envelhecem de forma saudável e conseguem manter níveis elevados de funcionamento cognitivo durante toda a vida (Antonini, et al., 2008; Dunnen, et al., 2008). Acresce que, em muitos casos, esse declínio não costuma ser pernicioso, isto é, não costuma ter consequências nocivas especialmente graves, que limitem irreparavelmente a funcionalidade ou a sobrevivência, de forma caracteristicamente patológica, mas, pelo contrário, ocorre em paralelo com mudanças fisiológicas, expectáveis em processos evolutivos normais. Todavia, em alguns indivíduos, as alterações de certas habilidades cognitivas ultrapassam aquilo que poderia ser considerado normal e apresentam-se como persistentemente progressivas. Essas formas perniciosas de deterioração intelectual podem ser causadas por diversas condições neuropatológicas, originando diferentes quadros demenciais.

"Demência" e "demente" são, na linguagem corrente, palavras estigmatizantes, evidenciando, de um modo geral, a falta de preparação para compreender e aceitar os males da mente (Pontes, 2007). Sob o ponto de vista clínico, a demência é considerada uma síndroma marcada pela ocorrência de múltiplos défices cognitivos ou de transtornos do funcionamento executivo. De acordo com o International Statistical Classification of Disease Related Health Problems da World Health Organization (2007), trata-se de uma situação em que se registam perturbações, mais ou menos acentuadas, da actividade social, profissional e até da vida quotidiana de indivíduos que, sem alteração do seu nível de consciência, manifestam alterações persistentes das funções nervosas superiores, especialmente da memória, do pensamento, da orientação, da aprendizagem, do cálculo, da compreensão e da capacidade para efectuar juízos críticos. Segundo os critérios apresentados pelo Diagnostic and Statistical Manual of Mental Disorders 
(American Psychiatric Association, 2000), demência é definida como um défice cognitivo múltiplo, afectando a memória e associando-se a, pelo menos, um de quatro sintomas de perturbação intelectual (afasia, agnosia, apraxia ou disfunção executiva), com declínio apreciável do prévio desempenho profissional ou social da pessoa, e que não ocorra no decurso de estado confusional ou de doença depressiva.

Assim, é crucial discriminar as situações de declínio cognitivo que acompanham o processo normal de envelhecimento, as dificuldades cognitivas associadas a condições psicopatológicas independentes da idade e as condições de declínio cognitivo pernicioso (Aarsland \& Ballard, 2004; Borroni, Agosti, \& Padovani, 2008; Guidi, Paciaroni, Paolini, Padova, \& Scarpino, 2006; Salmon \& Bondi, 2009). Para isso, pode contribuir de forma significativa, com base em métodos de diagnóstico diferencial, a avaliação neuropsicológica, realizada por equipas multidisciplinares que podem também contribuir de forma significativa para a estruturação de programas de reabilitação cognitiva, em qualquer um daqueles casos (Bertelli, et al., 2007).

Os psicólogos, em particular, podem desempenhar um papel de destaque na avaliação de queixas e de mudanças no funcionamento cognitivo, já que, graças ao seu conhecimento e treino na utilização de ferramentas especializadas, estão singularmente bem equipados para avaliar mudanças nos mecanismos de memória e nos processos de funcionamento e, ainda, para distinguir entre mudanças normais e sinais iniciais de patologia. Esse papel dos psicólogos não pode ser subestimado, uma vez que, apesar dos árduos esforços no sentido da identificação de causas fisiológicas de demência, ainda não existem, nem sequer para as formas mais comuns de demência, marcadores biológicos que permitam diagnósticos diferenciais conclusivos, salvo os indicadores "post mortem" que só podem ser obtidos, tarde demais, na autópsia (McKeith, 2007; Saver, Ffytche, Ballard, Brown, \& Howard, 2006).

As avaliações neuropsicológica e cognitiva representam os métodos de diagnóstico diferencial mais eficazes na discriminação entre o normal e o patológico, e entre as dificuldades cognitivas relacionadas com a depressão e outras disfunções e as condições de declínio cognitivo pernicioso (Bertelli, et al., 2007; Salmon \& Bondi, 2009). Entre as causas das condições de declínio cognitivo pernicioso está a DCL. Tal condição, apesar de representar entre $10 \%$ a $15 \%$ de todos os casos de demência (McKeith, Galasko, et al., 1996; McKeith, Mintzer, et al., 2004), é ainda pouco conhecida pelos próprios psicólogos. 
O conhecimento da $\mathrm{DCL}$, a segunda causa mais comum de demência neurodegenerativa em idosos (McKeith, 2005), é extremamente importante, uma vez que, devido às alucinações presentes nos estágios iniciais, pode ser interpretada como indicativa de um quadro psicopatológico, mais especificamente esquizofrenia, que traz como consequência imediata a prescrição de antipsicóticos ou neurolépticos, cujos efeitos colaterais, no caso da $\mathrm{DCL}$, são extremamente nocivos para o sistema extrapiramidal (Kane, 2001; Ray, et al., 2004).

O sistema piramidal conduz, a partir do córtex cerebral, os impulsos que possibilitam a execução de movimentos voluntários precisos. O sistema é composto por axónios que se originam nos corpos das células nervosas, ou neurónios, no córtex cerebral. É a única via cujos axónios ligam, sem a interrupção de quaisquer sinapses, o córtex cerebral aos seus destinos finais na medula espinhal. Esse sistema é chamado piramidal, devido ao formato de pirâmide dos corpos das células em que os axónios se originam e, também, para descrever uma área da superfície do eixo da medula oblonga, onde o sistema piramidal atravessa para o outro lado da medula espinhal. As vias extrapiramidais, por outro lado, são aquelas vias motoras que não passam através das pirâmides da medula oblonga (Greenstein \& Greenstein, 2000).

O sistema extrapiramidal consiste em vias centrais que regulam as áreas motoras do Sistema Nervoso Central, no córtex cerebral, no cerebelo, no tronco cerebral e na medula espinhal. A função primária do sistema extrapiramidal é a sintonia fina dos movimentos voluntários, no sentido de torná-los conscientemente reguláveis, em níveis mais elevados. A ausência dessa sintonia fina torna-se evidente em condições como o parkinsonismo, em que os movimentos voluntários são prejudicados, por exemplo, pela presença de tremores e abrandamento motor. Mesmo em doses baixas, indivíduos com DCL, que não apresentam alterações no sistema motor extrapiramidal, são extremamente susceptíveis a desenvolvê-las (por exemplo: movimentos espontâneos, rigidez, tremores), quando medicados com antipsicóticos (McKeith, 2006; McKeith, Mintzer, et al., 2004).

Em $75 \%$ a $80 \%$ dos casos de $\mathrm{DCL}$, observam-se sinais extrapiramidais (SEP), no curso natural da doença, porém, no momento do diagnóstico, esses sinais registam-se em apenas $25 \%$ a $50 \%$ dos casos, sugerindo que os SEP podem surgir ou agravar-se em consequência da medicação neuropsiquiátrica (McKeith, 2007). Essa é, por si só, razão bastante para realçar a importância da avaliação neuropsicológica e, portanto, do papel que os psicólogos podem desempenhar, contribuindo, por exemplo, para o diagnóstico diferencial entre condições 
neuropsiquiátricas e neurodegenerativas (McKeith \& Cummings, 2005; McKeith, Mintzer, et al., 2004).

Tem sido frequentemente sugerido que a DCL seja considerada parte de um espectro chamado Doença com Corpos de Lewy (Dodel, et al., 2008). Esse espectro abrange doenças neurodegenerativas que partilham o desequilíbrio e a acumulação anormal da proteína sináptica alfa-sinucleína (Spillantini, et al., 1997). As manifestações clínicas da Doença com Corpos de Lewy incluem a DCL, a Doença de Parkinson (DP) e falhas no Sistema Nervoso Autónomo que, em todos os casos referidos, incluem a formação de corpos de Lewy ou de neuritos de Lewy (Emre, 2003; Popescu \& Lippa, 2004).

Os corpos de Lewy e os neuritos de Lewy são depósitos microscópicos de proteínas. Enquanto os corpos de Lewy se apresentam como "saquinhos", dentro da célula cerebral, os neuritos de Lewy têm a forma de "fitinhas", nos prolongamentos neuronais. Friederich Heinrich Lewy descobriu, no cérebro de indivíduos com DP, uma formação ínfima, que posteriormente passou a chamar-se corpo de Lewy. O termo corpos de Lewy entrou na literatura internacional em 1918 (Schiller, 2000).

As proteínas são substâncias que têm a função de libertar energia e de reparar as células e os tecidos. A proteína alfa-sinucleína, em particular, habitualmente protege as células, mas a sua acumulação leva à formação de corpos de Lewy (Spillantini, et al., 1997). Ainda não ficou esclarecido se os corpos de Lewy e os neuritos de Lewy são a causa ou a consequência da degeneração das células cerebrais. As causas dos sintomas clínicos parecem surgir a partir de deficiências de neurotransmissão e dos efeitos desorganizadores dos corpos de Lewy sobre as funções dos axónios (Mukaetova-Ladinska \& McKeith, 2006). É oportuno observar que não parecem existir factores genéticos implicados no processamento patológico da proteína sináptica alfa-sinucleína, nos casos de Doença com Corpos de Lewy (Johnson, et al., 2004; McKeith, 2006, 2007; McKeith, Mintzer, et al., 2004).

Muitos casos de DCL também apresentam, na autópsia, algumas características da Doença de Alzheimer (DA), predominantemente na forma de placas amilóides (uma proteína sináptica complexa que resulta da degeneração do tecido). Emaranhados neurofibrilares no neocórtex ocorrem em $20 \%$ dos casos e a presença desses emaranhados modifica a apresentação clínica típica da $D C L$, tornando-a semelhante à da DA. Isso dificulta a distinção dos casos, considerando-se apenas a apresentação clínica, porém a identificação exacta da $D C L$, que não deixa de ser 
possível, já que indivíduos com DCL apresentam sintomas específicos, deficiências e incapacidades que diferem dos apresentados por indivíduos com DA, é particularmente importante, pois, como se viu, as confusões de diagnóstico podem ter graves consequências, pelo efeito perverso das terapêuticas instituídas erroneamente (McKeith, 2005; Tiraboschi, et al., 2006).

O consenso para os critérios diagnósticos clínicos de provável $D C L$ ou de possível DCL (McKeith, 2006; McKeith \& Cummings, 2005; McKeith, Galasko, et al., 1996; McKeith, Mintzer, et al., 2004) especifica um conjunto de características centrais, com manifestação saliente: a) um declínio cognitivo progressivo, de tal magnitude que interfira com as funções sociais e ocupacionais normais; $b$ ) danos persistentes na memória, que não ocorrem necessariamente nos estágios iniciais, mas que são evidentes com a evolução do quadro na maioria dos casos; c) défices de atenção.

As características basilares (duas delas essenciais para o diagnóstico de provável DCL e uma delas para o diagnóstico de possível $D C L$ ) são: a cognição irregular com variações pronunciadas na atenção e na prontidão para responder; alucinações visuais repetidas, tipicamente bem formadas e detalhadas; e características espontâneas da DP. Há ainda três características sugestivas de DCL (McKeith, 2006, 2007), cuja ocorrência, isolada ou em combinação, num indivíduo com demência, dão suporte adicional ao diagnóstico de DCL: desordem comportamental de sono REM (rapid eye movement), extrema sensibilidade aos efeitos colaterais dos antipsicóticos e baixa captação de dopamina pelos glânglios basais, conforme ilustrado pela tomografia computadorizada por emissão de fótão único (SPECT) ou pela tomografia por emissão de positrões (PET) (Boeve, Silber, \& Ferman, 2004; McKeith, O'Brien, et al., 2007). Existem ainda algumas características auxiliares, ou seja, que podem estar presentes, mas que não constituem os elementos mais importantes: quedas repetidas, desmaios, breves perdas de consciência, ilusões sistematizadas, alucinações em outros domínios que não o visual (por exemplo, alucinações auditivas) e depressão.

A especificidade de um diagnóstico clínico de provável $\mathrm{DCL}$, definido pela presença de duas, ou mais, das características centrais, representa um critério exigente em mais de $80 \%$ dos casos, que, segundo McKeith (2007), só é seguido exclusivamente em cerca de metade dos casos. Na prática clínica, para melhorar a taxa de identificação de casos, utiliza-se um índice mais elevado de suspeita, adoptando como critério, no diagnóstico clínico de possível $\mathrm{DCL}$, a presença de uma única característica central. 
O conhecimento e o reconhecimento de uma provável $D C L$ é particularmente relevante, considerando-se o significativo benefício oferecido pela farmacoterapia, através, por exemplo, de drogas que aumentem a disponibilidade de acetilcolina, isto é, do neurotransmissor que assegura a transmissão dos estímulos nervosos, na fenda sináptica, entre células do sistema colinérgico (Cummings, 2000; McKeith, Ser, et al., 2000; Perry, et al., 2000). Além disso, torna-se essencial a estruturação de programas de reabilitação cognitiva direccionados especificamente para os domínios do funcionamento cognitivo mais comprometidos (Ballard, et al., 2002).

Muitas similaridades clínicas e patológicas entre a $D C L$ e a Demência que ocorre nos estágios mais avançados da Doença de Parkinson (DDP) têm sido reconhecidas. Até mesmo na autópsia, indivíduos com DCL e DDP apresentam corpos de Lewy, que constituem a característica patológica da $D C L$, no entanto, DCL e DDP são síndromes clinicamente definidas (McKeith \& Cummings, 2005; McKeith, Galasko, et al., 1996; McKeith, Mintzer, et al., 2004).

A sequência temporal dos eventos é bastante importante, porque, sabe-se que, quando $\mathrm{O}$ indivíduo apresenta apenas sinais motores, o problema será plausivelmente DP, mas, quando os sinais motores são seguidos (dentro de um período de 12 meses) por sinais cognitivos, é mais plausível que o problema seja $\mathrm{DCL}$, no entanto, quando os sinais motores são seguidos (depois de mais de 12 meses do seu aparecimento) por sinais cognitivos, o problema plausivelmente será DDP, que tende a manifestar-se na fase mais avançada da doença (McKeith, 2006).

Geralmente, o princípio da DCL tende a ser muito discreto, de difícil detecção, sem que o próprio indivíduo ou os outros à sua volta percebam que algo ocorre, no entanto, esse começo é destrutivo. A presença de um período de crescente confusão, a identificação da presença de alucinações ou uma queda significativa, podem dar a falsa impressão de que o início foi repentino, fulminante. $O$ desenrolar da $\mathrm{DCL}$, no entanto, é progressivo, com a pontuação nas avaliações cognitivas declinando cerca de 10\%, por ano. As flutuações cognitivas podem contribuir para a elevada variabilidade apresentada em termos das pontuações obtidas no MiniExame do Estado Mental (MEEM), o que dificulta a determinação do grau de gravidade do prejuízo em uma única avaliação (McKeith, 2007).

Os principais diagnósticos diferenciais para a $\mathrm{DCL}$ são a $\mathrm{DA}$, a demência vascular, a DDP, as síndromes Parkinsonianas atípicas (por exemplo, paralisia supranuclear progressiva, atrofia sistémica múltipla, degeneração córticobasal e a doença de Creutzfeldt-Jacob). O tempo de sobrevivência, a partir do início da $\mathrm{DCL}$ até à morte, é geralmente similar ao da DA, muito embora, para alguns indivíduos com 
DCL, o curso da doença seja bem mais rápido (McKeith, 2007; McKeith, Galasko, et al., 1996).

O diagnóstico clínico da $\mathrm{DCL}$ depende da obtenção de uma história detalhada de sintomas, a partir de entrevistas com o próprio indivíduo e algum informador próximo do mesmo, do exame do estado mental e das avaliações neurológica e cognitiva apropriadas. Investigações através de recursos de neuroimagem podem dar suporte ao diagnóstico clínico (Saver, et al., 2006). As mudanças associadas à DCL incluem a preservação do volume do hipocampo e do lobo temporal medial, segundo MRI, e diminuição do fluxo sanguíneo na região occipital, segundo SPECT, perda do transportador de dopamina no caudato e no putámen como consequência de degeneração das vias dopaminérgicas nigroestriatais (McKeith, O'Brien, et al., 2007).

O perfil de danos neuropsicológicos na $\mathrm{DCL}$ reflecte o envolvimento combinado das vias corticais e subcorticais e a relativa preservação do hipocampo. Os indivíduos com $\mathrm{DCL}$ apresentam um desempenho pobre em tarefas visuoespaciais e testes de atenção (Sauer, et al., 2006). As flutuações no funcionamento cognitivo, que podem alterar-se em minutos, horas ou dias, ocorrem em $50 \%$ a $75 \%$ dos casos e estão associadas à contínua variação dos níveis de atenção e prontidão para responder (McKeith, 2007). Os outros, à sua volta, observam a presença de sonolência durante o dia, os episódios em que o indivíduo fica olhando fixamente, sem expressão, para o nada e ainda a fala incoerente (Ferman, et al., 2004; McKeith, 2006).

As alucinações visuais são o traço neuropsiquiátrico mais característico da DCL e a sua persistência ajuda no diagnóstico diferencial. As alucinações visuais estão presentes em metade dos casos de $\mathrm{DCL}$, no momento em que a doença se apresenta, e ocorrem, em algum momento durante o curso da doença, na maioria dos casos. Essas alucinações consistem em imagens muito bem formadas, detalhadas e animadas, que geram respostas emocionais variadas e estão associadas à deficiência de acetilcolina cortical (Salmon \& Bondi, 2009).

O perfil de SEP na DCL é geralmente similar ao perfil de SEP nos casos de DP, porém com maior instabilidade postural, apatia facial e menos tremor. Uma sensibilidade anormal aos efeitos colaterais da medicação antipsicótica, conforme já referido, ocorre em $50 \%$ a $60 \%$ dos casos de $\mathrm{DCL}$, em que são receitados agentes antipsicóticos tradicionais, e essa sensibilidade severa está associada a um aumento significativo do risco de mortalidade, devido ao início intenso ou agravamento dos SEP e de danos da consciência (Kane, 2001; MacKeith, 2007). Os 
antipsicóticos atípicos, utilizados em pequenas doses, são mais seguros nesse sentido, mas as reacções de sensibilidade têm sido documentadas com a maioria desses agentes (McKeith, Mintzer, et al., 2004).

Uma perturbação frequentemente associada à $D C L$, à DDP e à atrofia sistémica múltipla, mas que raramente ocorre em outros quadros demenciais, e que pode contribuir para as alucinações e alterações comportamentais típicas da DCL, é a parassónia do sono paradoxal (REM), caracterizada por pesadelos, vívidos e aterrorizadores, e por interrupções, mais ou menos bruscas, da atonia motora que normalmente se observa na fase do sono dita paradoxal, por nela a actividade e o metabolismo estarem aumentados e se observar a supressão do tónus muscular, ou REM, por nela se registarem movimentos oculares rápidos (Boeve, et al., 2004; Dodel, et al., 2008).

Sintomas de depressão (desinteresse por todas, ou quase todas, as formas de actividade, durante todo o dia e quase todos os dias, perda de peso, na ausência de dieta, aumento ou diminuição do apetite, quase todos os dias, distúrbios do sono, falta de energia, quase todos os dias, sentimentos de inferioridade ou sentimentos de culpa, diminuição da capacidade de concentração) são relatados em 33\% a 50\% dos casos de DCL (McKeith, 2007).

Como referido no início, a revisão que agora se conclui visou oferecer uma panorâmica básica da Demência de Corpos de Lewy ( $D C L)$. Com esse intuito, foram dados, sucessivamente, os passos seguintes.

Pretendeu-se destacar que é tão importante reconhecer, identificar e tratar, ou acompanhar, as patologias que possam afectar pessoas com mais idade, quanto não perder de vista que o processo de envelhecimento se faz acompanhar por mudanças que, mesmo quando se traduzam em certas limitações das capacidades ou da actividade, não são necessariamente anormais e não devem ser confundidas com manifestações patológicas.

Procedeu-se à caracterização genérica da demência e ressaltou-se a necessidade de equipas multidisciplinares procederem à demarcação entre as situações de declínio cognitivo que acompanham o processo normal de envelhecimento, as dificuldades cognitivas associadas a condições psicopatológicas independentes da idade e as condições de declínio cognitivo pernicioso.

Salientou-se, em particular, a relevância do papel dos psicólogos, quer pela importância das avaliações neuropsicológica e cognitiva, para o diagnóstico 
diferencial dos quadros demenciais, quer pela responsabilidade que lhes deve ser atribuída no desenho e concretização de programas de reabilitação cognitiva.

Relembrou-se a posição nuclear que a DCL ocupa, no conjunto das patologias demenciais mais frequentes entre os idosos, como segunda causa mais comum de demência neurodegenerativa, com uma prevalência de 10\% a 15\% dos casos pertinentes.

Referiu-se o risco de confusão, no diagnóstico, entre a DCL e outras patologias, e a gravidade das consequências eventualmente resultantes, especialmente as associadas ao uso erróneo de antipsicóticos ou neurolépticos.

Por fim, caracterizou-se abreviadamente o quadro etiológico da DCL e sistematizou-se o conjunto de sinais e de sintomas que podem estabelecer o seu diagnóstico seguro, provável ou possível.

\section{Conclusões}

Dada a sua máxima relevância, face aos objectivos deste artigo, conclui-se, retomando, de modo esquemático, as linhas de orientação para o diagnóstico diferencial da DCL:

1. Características centrais (essenciais para o diagnóstico de possível ou provável DCL):

Declínio cognitivo progressivo com magnitude suficiente para interferir com funções sociais ou ocupacionais normais;

Défice proeminente ou persistente da memória pode não ocorrer necessariamente nos estágios iniciais, mas é normalmente evidente com o progresso;

Défices nos testes de atenção, função executiva e habilidade visuoespacial podem ser especialmente proeminentes;

2. Características basilares (duas características basilares são suficientes para um diagnóstico de provável $D C L$ e uma para o de possível $D C L$ ):

Cognição flutuante com variações pronunciadas na atenção e na vigília;

Alucinações visuais recorrentes que são tipicamente bem formadas e detalhadas;

Características espontâneas da DP;

3. Características sugestivas (se uma ou mais estiver presente em conjunto com uma ou mais características basilares, pode fazer-se um diagnóstico de provável $\mathrm{DCL}$; na ausência de qualquer característica basilar, uma ou mais característica 
sugestiva é suficiente para possível $D C L$; o diagnóstico de provável $D C L$ não deverá ser feito com base exclusivamente em características sugestivas):

Parassónia do sono paradoxal;

Sensibilidade neuroléptica severa;

Baixa captação do transportador de dopamina nos gânglios basais demonstrada por imagens SPECT ou PET;

4. Características auxiliares (habitualmente presentes, mas que não se provou que tenham especificidade diagnóstica):

Quedas repetidas e síncope;

Perda transitória e inexplicada de consciência;

Disfunção autonómica severa, por exemplo, hipotensão ortoestática ou incontinência urinária;

Alucinações em outras modalidades, que não a visual;

Depressão;

Relativa preservação das estruturas do lobo temporal medial conforme TC (tomografia computadorizada) / MR (ressonância magnética);

5. Um diagnóstico de $\mathrm{DCL}$ é menos provável:

Na presença de doença cerebrovascular evidente como sinais neurológicos focais ou em neuroimagem;

Na presença de qualquer outra doença física ou disfunção cerebral para dar conta total ou parcialmente do quadro clínico;

Se o parkinsonismo somente aparece pela primeira vez no estágio de demência severa;

6. Sequência temporal dos sintomas:

DCL deveria ser diagnosticada quando ocorre demência antes ou concomitantemente com parkinsonismo (se este estiver presente);

O termo Demência na doença de Parkinson (DDP) deve ser usado para descrever a demência no contexto de doença de Parkinson bem estabelecida;

Num contexto prático, termos genéricos como Doença com Corpos de Lewy são frequentemente úteis;

Em estudos recentes em que há necessidade de distinguir entre $D C L$ e DDP, a regra de um ano na instalação da demência e do parkinsonismo na $\mathrm{DCL}$ continua a ser recomendada. A adopção de outros períodos de tempo irá simplesmente confundir a confrontação de dados e a comparação de estudos. Em outros contextos de investigação que 
60 | Revisão para psicólogos sobre demência de corpos de Lewy

podem incluir estudos clinicopatológicos e ensaios clínicos, ambos os fenótipos clínicos podem ser considerados em conjunto sob categorias tais como a Doença com Corpos de Lewy ou Alfa-sinucleinopatias.

\section{Bibliografia}

Aarsland, D., \& Ballard, C. (2004). Psychiatric issues in non-Alzheimer dementias. Clinical Neuroscience Research, 3, 397-412.

American Psychiatric Association. (2000). Diagnostic and statistical manual of mental disorders (text revision). Washington, DC: Author.

Antonini, F.M., Magnolfi, S.U., Petruzzi, E., Pinzani, P., Malentacchi, F., Petruzzi, I., et al. (2008). Physical performance and creative activities of centenarians. Archives of Gerontology and Geriatrics, 46, 253-261.

Ballard, C.G., Court, J.A., Piggott, M., Johnson, M., O'Brien, J., McKeith, I., et al. (2002). Disturbances of consciousness in dementia with Lewy bodies associated with alteration in nicotinic receptor binding in the temporal cortex. Consciousness and Cognition, 11, 461-474.

Bertelli, R., Vasconcelos-Raposo, J., \& Fernandes, H. (2007, setembro). Avaliação neuropsicológica e estruturação de programas de reabilitação cognitiva para o idoso. Comunicação apresentada na Conferência Novos desafios da institucionalização de idosos: combate à solidão acompanhada, Lamego, Portugal.

Boeve, B.F., Silber, M.H., \& Ferman, T.J. (2004). REM sleep behavior disorder in Parkinson's disease and Dementia with Lewy bodies. Journal of Geriatric Psychiatry and Neurology, $17,146-157$.

Borroni, B., Agosti, C., \& Padovani, A. (2008). Behavioral and psychological symptoms in dementia with Lewy-bodies (DLB): Frequency and relationship with disease severity and motor impairment. Archives of Gerontology and Geriatrics, 46, 101-106.

Cummings, J.L. (2000). Cholinesterase inhibitors: Expanding applications. The Lancet, 356(16), 2024-2025.

Dodel, R., Csoti, I., Ebersbach, G., Fuchs, G., Hahne, M., Kuhn, W., et al. (2008). Lewy body dementia and Parkinson's disease with dementia. Journal of Neurology, 255(5), 39-47.

Dunnen, W.F.A., Brouwer, W.H., Bijlard, E., Kamphuis, J., Linschoten, K., Eggens-Meijer, E., et al. (2008). No disease in the brain of a 115-year-old woman. Neurobiology of Aging, 29, 1127-1132.

Emre, M. (2003). Dementia associated with Parkinson's disease. Lancet Neurology, 2, 229237.

Greenstein, B., \& Greenstein, A. (2000) Color atlas of neuroscience: Neuroanatomy and neurophysiology. Stuttgart: Thieme.

Guidi, M., Paciaroni, L., Paolini, S., Padova, S., \& Scarpino, O. (2006). Differences and similarities in the neuropsychological profile of dementia with Lewy bodies and 
Alzheimer's disease in the early stage. Journal of the Neurological Sciences, 248, 120123.

Johnson, J., Hague, S.M., Hanson, M., Gibson, A., Wilson, K.E., Evans, E.W., et al. (2004). SNCA multiplication is not a common cause of Parkinson disease or dementia with Lewy bodies. Neurology, 63(3), 554-556.

Kane, J.M. (2001). Extrapiramidal side effects are unacceptable. European Neuropsychopharmacology, 11(4), S397-S403.

McKeith, I. (2005). Dementia with Lewy bodies. Psychiatry, 4(1), 52-55.

McKeith, I. (2005). What's new in Psychiatry: Dementia with Lewy bodies. Medicine, 33(3), 14 .

McKeith, I. (2006). Consensus guidelines for the clinical and pathologic diagnosis of dementia with Lewy bodies (DLB): Report of the Consortium on DLB International Workshop. Journal of Alzheimer's Disease, 9, 417-423.

McKeith, I. (2007). Dementia with Lewy bodies. Psychiatry, 7(1), 20-23.

McKeith, I., \& Cummings, J. (2005). Behavioural changes and psychological symptoms in dementia disorders. Lancet Neurology, 4, 735-742.

McKeith, I., Mintzer, J., Aarsland, D., Burn, D., Chiu, H., Cohen-Mansfield, J., et al. (2004). Dementia with Lewy bodies. Lancet Neurology, 3, 19-28.

McKeith, I., O'Brien, J., Walker, Z., Tatsch, K., Booij, J., Darcourt, J. et al., (2007). Sensitivity and specificity of dopamine transporter imaging with 123 l-FP-CIT SPECT in dementia with Lewy bodies: a phase III, multicentre study. Lancet Neurology, 6, 305-313.

McKeith, I., Ser, T., Spano, P., Emre, M., Wesnes, K., Anand, R., et al. (2000). Efficacy of rivastigmine in dementia with Lewy bodies: A randomised, double-blind, placebocontrolled international study. The Lancet, 356(16), 2031-2036.

McKeith, I.G., Galasko, D., Kosaka, K., Perry, E.K., Dickson, D.W., Hansen, L.A., et al. (1996). Dementia with Lewy bodies: diagnostic criteria and pathological guidelines. Report of the consortium on dementia with Lewy bodies. Neurology, 47, 1113-1124.

Mukaetova-Ladinska, E.B., \& McKeith, I.G. (2006). Pathophysiology of synuclein aggregation in Lewy bodies disease. Mechanisms of Ageing and Development, 127, 188202.

Perry, A., Martin-Ruiz, C., Lee, M., Griffiths, M., Johnson, M., Piaggot, M., et al. (2000). Nicotinic receptor subtypes in human brain ageing, Alzheimer and Lewy bodies diseases. European Journal of Pharmacology, 393, 215-222.

Pontes, C. (2007). Perspectiva neurológica sobre as demências. Revista da Faculdade de Ciências da Saúde, 4, 36-43.

Popescu, A., \& Lippa, C.F. (2004). Parkinsonian syndromes: Parkinson's disease dementia, dementia with Lewy bodies and progressive supranuclear palsy. Clinical Neuroscience Research, 3, 461-468.

Ray, M., Bohr, I., McIntosh, J.M., Ballard, C., McKeith, I., \& Chalon, S. (2004). Involvement of alpha6/alphaz neuronal nicotinic acetylcholine receptors in neuropsychiatric features 
of Dementia with Lewy bodies: (125l)-alpha- conotoxin MII binding in the thalamus and striatum. Neuroscience Letters, 372, 220-225.

Salmon, D.P., \& Bondi, M.W. (2009). Neuropsychological assessment of dementia. Annual Review of Psychology, 60, 257-282.

Sauer, J., Ffytche, D.H., Ballard, C., Brown, R.G., \& Howard, R. (2006). Differences between Alzheimer's disease and dementia with Lewy bodies: An fMRI study of task-related brain activity. Brain, 129, 1780-1788.

Schiller, F. (2000). Fritz Lewy and his bodies. Journal of the History of the Neurosciences, $9(2), 148-151$.

Spillantini, M.G., Schmidt, M.L., Lee, V.M.Y., Trojanowski, J.Q., Jakes, R., \& Goedert, M. (1997). Alpha-Synuclein in Lewy bodies. Nature, 388, 839-840.

Tiraboschi, P., Salmon, D.P., Hansen, L.A., Hofstetter, R.C., Thal, L.J., \& Corey Bloom, J. (2006). What best differentiates Lewy body from Alzheimer's disease in early-stage dementia? Brain, 129, 729-735.

World Health Organization. (2007). International Statistical Classification of Diseases and Related Health Problems (1oth revision). Geneva: Author. 